\title{
Research on Ubiquitous Map Information Organization Oriented to Location-Based Aggregation
}

\author{
Si Wang, Guangxia Wang \\ Institute of Geographical Spatial Information, Information Engineering University, Zhengzhou, China \\ Email: plaieu_wangs@163.com
}

How to cite this paper: Wang, S., \& Wang, G. X. (2022). Research on Ubiquitous Map Information Organization Oriented to Location-Based Aggregation. Journal of Geoscience and Environment Protection, 10, 238-249.

https://doi.org/10.4236/gep.2022.101016

Received: January 11, 2022

Accepted: January 26, 2022

Published: January 30, 2022

\begin{abstract}
In the era of information and communication technology (ICT) and big data, the map gradually shows a new qualitative feature of "spatiotemporal ubiquitous" due to the extension of its object space and the geographic information it contains, which brings new challenges to map information organization. This paper analyzes the concept and information characteristics of the ubiquitous map. Based on that, it proposes a ubiquitous map information organization model oriented to location-based aggregation. This new model includes three parts as "ubiquitous map instance", "location-based aggregation mode" and "map scene". This paper focuses on the "map scene" part which is the core of the model and contains two mutually mapped aspects as "content scene" and "representation scene". And both aspects are divided into three levels as "features" $\leftrightarrow$ "elements" $\leftrightarrow \rightarrow$ "scenes" according to ubiquitous map information characteristics and location-based aggregation mode. With cases of map decomposition, the application of the model is explained to illustrate its effectiveness. The model is expected to provide powerful data organization and management capabilities for ubiquitous map production and use.
\end{abstract}

\section{Keywords}

Ubiquitous Map, Information Organization, Location-Based Aggregation, Map Scene

\section{Introduction}

Map information organization plays an important role in the production and use of maps and has always been one of the core contents of cartographic research. Oriented to a specific space, map information organization is to achieve 
the orderly, high-quality and scientific combination of information in the map space through the selection, analysis, description and sorting of the external and content features of geographic information using the scientific rules and methods of map construction. Traditional maps are oriented to the dual space of "geography-humanity". In terms of information organization, they are mainly based on the precise spatial positioning of geographic elements to collect and associate related geometric and attribute features, and represent them with two-dimensional graphic symbols, colors, characters and other external features, forming simple scenes with the main content of "spatial distribution of specific geographic elements" and the main representation of "two-dimensional, static, graphical and complete map structure". Entering the era of ICT (information and communication technology) and big data, maps gradually show a trend of "ubiquitous" development (Liu, Guo, Guo, Gao, \& Cui, 2020): on the one hand, the object and expression space of maps has been expanded from the traditional dual space of "geography-humanity" to the ternary space of "geography-humanity-information" (Guo, Chen, Ying, Lv, \& Li, 2018; Zhou, 2015), in which the join of information space has greatly expanded the object content and representation of maps; on the other hand, the information source of maps has been expanded from standard geographic information to fragmented and multi-modal spatiotemporal ubiquitous information (Kresse \& Danko, 2012), making the semantic content of maps increasingly refined and enriched. New changes and expansions make the ubiquitous map information organization face complex, diverse and dynamic scenes, for which the traditional map information organization model cannot provide sufficient support.

Location-based aggregation is a solution proposed in the era of ICT and big data to solve the contradiction between the massive spatiotemporal ubiquitous information and the complex information needs of users in the ternary space. It is an organic combination of the method of "information aggregation" (Cao \& $\mathrm{Ma}, 2016)$ in the field of information organization and retrieval and the concept of "panoramic location-based map" (Zhou, Zhu, Wang, Shi, \& Ou, 2011; Zhu, Zhou, Guo, Hu, Liu, \& Gao, 2015) in the field of cartography. And its core is to filter, aggregate, associate and fuse fragmented and multi-modal spatiotemporal ubiquitous information based on the semantic location, and dynamically generate application scenes to meet users' needs. Location-based aggregation can provide users with accurate location-related knowledge services, and has become an important application mode of spatiotemporal ubiquitous information. The consistency in coverage, information sources, and basic frameworks makes location-based aggregation an effective mode to provide support for the orderly and scientific organization of ubiquitous map information.

In view of the above, this paper introduces the idea of "location-based aggregation" into ubiquitous map information organization and establishes a ubiquitous map information organization model, which expands the traditional map information organization model in terms of both content and representation to form a complete ubiquitous map information organization mechanism. 
It is hoped that the research of this paper could help promote the automatic construction of ubiquitous maps and the effective use of map information.

\section{Basic Concept and Characteristics}

\subsection{Ubiquitous Map}

The ubiquitous map is the extension and expansion of the traditional map, and it is a comprehensive expression of the ternary space of "geography-humanityinformation". It uses map language, image thinking and spatial thinking to analyze the characteristics of ternary space objects and realize the functions of information acquisition, transmission, and cognition between people and people, people and things, and things and things (Guo, Chen, Ying, Lv, \& Li, 2018).

The ternary space constitutes a complex giant system. With the support of advanced observation technology, it has created a vast sea of spatiotemporal data streams and constituted a new and diverse map expression space (Ai, 2016). Facing the ternary space, the ubiquitous map breaks through the constraints of the traditional physical space, able to represent a variety of contents in a variety of ways and with new changes in many aspects compared with the traditional map, showing obvious "ubiquitous" characteristics. In this regard, scholars have given analysis and explanations from different perspectives and levels. They have proposed new concepts of "pseudo map" (Meng, 2017), "schematic map" (Liu \& Li, 2016), "quasi-map" (Guo \& Ying, 2017), "pan-map" (Guo, Chen, Ying, Lv, \& Li, 2018), and "we-map" (Yan, Zhang, Du, Liu, Wang, \& Yang, 2016). These can all be included in the concept of "ubiquitous map". In reality, there are various specific types of ubiquitous maps that are widely used, such as: cyber maps, virtual reality maps, augmented reality maps, mixed reality maps, semantic maps, metaphor maps, exaggerated deformation maps, metro roadmaps, 3D city maps, personalized mobile maps, etc.

For the ubiquitous map, the addition of the information space expands a variety of new qualitative expressing contents, such as: network events, processes and phenomena in the virtual space; semantic information of the objects in the collective space. Compared with the traditional map's element organization mode based on precise geographic positioning, the ubiquitous map describes and organizes ternary space objects based on the semantic location framework with richer content. On the other hand, the addition of the information space also expands more abundant means of expression for the ubiquitous map. The successive introduction of visual, auditory, tactile, temporal, spatial, virtual, and interactive variables enriches the representation forms of the ubiquitous map, and the design of its mathematical basis and symbol system is more flexible. For example, on the premise of ensuring the scientific nature of the map, its surface is no longer limited to the orthographic projection plane; it allows multiple mathematical conversion relations between the map coordinates and the geographic coordinates; it allows multiple scales to exist in the same map; and the graphic elements on it are no longer limited to vector symbols (Meng, 2017). 


\subsection{Information Characteristics of the Ubiquitous Map}

In essence, both ubiquitous maps and traditional maps are abstract descriptions and expressions of space supported by map synthesis, map projection and map visualization (Wang, Wang, \& Tian, 2021). And they both have the ability to transmit, organize and express geographic information. The difference is that ubiquitous maps have further expansions in information dimension, granularity, hierarchy, expression and semantics against the background of spatiotemporal big data (Wang, 2017; Liu, Fang, Guo, \& Gao, 2014), showing a more all-encompassing connotation and characteristics. This paper gives a summary for this in Table 1.

\section{Ubiquitous Map Information Organization Model Oriented to Location-Based Aggregation}

According to the information characteristics of the ubiquitous map, this paper introduces the mode and concept of location-based aggregation, and establishes a hierarchical, structured and systematic ubiquitous map information organization model (Figure 1). The model consists of three parts as "ubiquitous map instance", "location-based aggregation mode" and "map scene". Among them, the "ubiquitous map instance" part is a collection of various ubiquitous maps, which not only displays the results of ubiquitous map information organization,
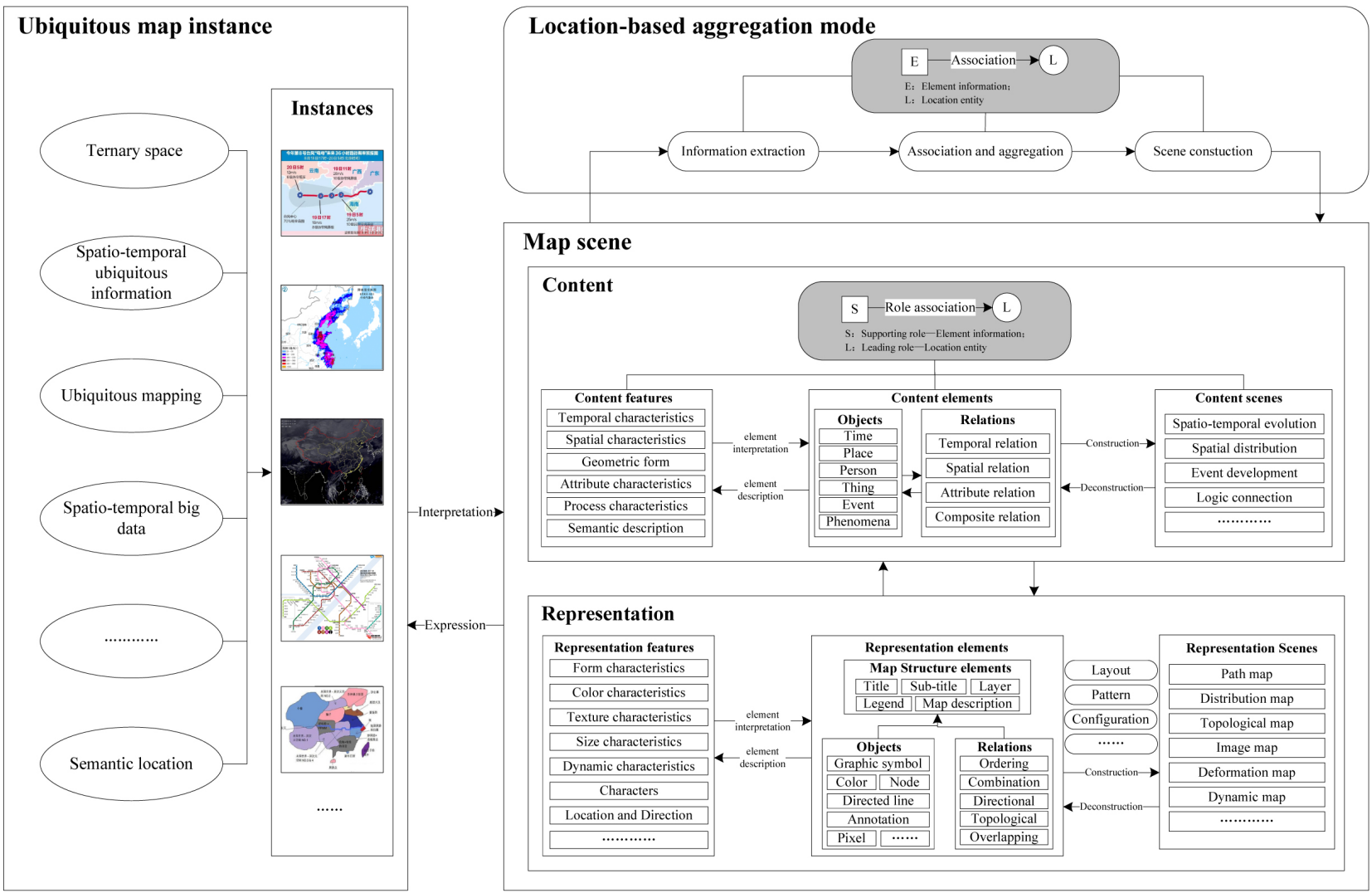

Figure 1. Ubiquitous map information organization model oriented to location-based aggregation. 
Table 1. Comparison between the ubiquitous map and the traditional map.

\begin{tabular}{|c|c|c|c|}
\hline \multirow{2}{*}{\multicolumn{2}{|c|}{ Comparison Items }} & \multicolumn{2}{|c|}{ Map Type } \\
\hline & & Traditional map & Ubiquitous map \\
\hline \multirow{4}{*}{$\begin{array}{l}\text { role orientation } \\
\text { of the map and } \\
\text { its information }\end{array}$} & nature & $\begin{array}{l}\text { Objective product based on scientific } \\
\text { observation }\end{array}$ & $\begin{array}{l}\text { Social product in the ICT and } \\
\text { big data environment }\end{array}$ \\
\hline & object space & Geographical and social spaces & $\begin{array}{l}\text { A hybrid of geographical, } \\
\text { social and information spaces }\end{array}$ \\
\hline & use & $\begin{array}{l}\text { Popularized spatial cognition and } \\
\text { generalized industry application }\end{array}$ & More for personalized applications \\
\hline & creators/providers & Professionals & $\begin{array}{l}\text { Professionals, amateurs, users, } \\
\text { volunteers, and even everyone }\end{array}$ \\
\hline \multirow{4}{*}{$\begin{array}{l}\text { information } \\
\text { organization } \\
\text { of map surface }\end{array}$} & $\begin{array}{l}\text { composition and } \\
\text { layout of map surface }\end{array}$ & $\begin{array}{l}\text { Complete composition of the main body, } \\
\text { title, legend, etc.; uniform and } \\
\text { standardized layout }\end{array}$ & $\begin{array}{l}\text { All components other than the } \\
\text { main body may default; flexible } \\
\text { and diverse layout }\end{array}$ \\
\hline & basic framework & Spatiotemporal coordinate & Semantic location \\
\hline & $\begin{array}{l}\text { information } \\
\text { processing }\end{array}$ & $\begin{array}{l}\text { Professional processing according to } \\
\text { strict and clear standards }\end{array}$ & $\begin{array}{l}\text { Professional or non-professional } \\
\text { processing with or without standards }\end{array}$ \\
\hline & $\begin{array}{l}\text { spatiotemporal } \\
\text { accuracy }\end{array}$ & Generally high & Possibly high or low \\
\hline \multirow{3}{*}{$\begin{array}{l}\text { data source } \\
\text { of map } \\
\text { information }\end{array}$} & nature & Spatial data and thematic data & Spatiotemporal big data \\
\hline & characteristics & $\begin{array}{l}\text { Relatively single source, mainly structured, } \\
\text { single point positioning, static, with } \\
\text { weak data currency and long update cycle }\end{array}$ & $\begin{array}{l}\text { Multi-source, heterogeneous, } \\
\text { multidimensional, dynamic, with } \\
\text { strong data currency and short } \\
\text { update cycle (near real-time update) }\end{array}$ \\
\hline & $\begin{array}{l}\text { acquisition } \\
\text { methods }\end{array}$ & $\begin{array}{l}\text { Traditional surveying and mapping, } \\
\text { applying according to user authority, } \\
\text { with high cost }\end{array}$ & $\begin{array}{l}\text { Intelligent and socialized ubiquitous } \\
\text { surveying and mapping, with low cost }\end{array}$ \\
\hline \multirow{4}{*}{$\begin{array}{l}\text { content of map } \\
\text { information }\end{array}$} & breadth & Focused on the geospatial pattern & $\begin{array}{l}\text { Synthesis of natural, human and } \\
\text { social aspects; involving multiple } \\
\text { scales, multiple dimensions, multiple } \\
\text { attributes, and multiple spaces }\end{array}$ \\
\hline & depth & Mainly shallow processing information & $\begin{array}{l}\text { Involving raw information } \\
\text { (observation data), shallow processing } \\
\text { information and deep processing } \\
\text { information (knowledge and laws) }\end{array}$ \\
\hline & levels of detail & $\begin{array}{l}\text { Focused on spatiotemporal location, } \\
\text { geometric structure and attribute } \\
\text { characteristics }\end{array}$ & $\begin{array}{l}\text { Equally emphasized on spatial } \\
\text { location, geometric form, attribute } \\
\text { characteristics, objects relationship, } \\
\text { evolution process, and semantic } \\
\text { description }\end{array}$ \\
\hline & completeness & $\begin{array}{l}\text { Large-scale and comprehensive contents } \\
\text { around the map theme }\end{array}$ & $\begin{array}{l}\text { Can be fragmented } \\
\text { "micro-contents" }\end{array}$ \\
\hline
\end{tabular}


but also provides data support for the reorganization of ubiquitous map information. The "location-based aggregation mode" part reflects the process and principle of location-based aggregation, and is the method basis and mode support for the organization of ubiquitous map information. The "map scene" part reflects the hierarchical organization mechanism and law of ubiquitous map information from internal content to external representation under the location-based aggregation mode, which is the core of ubiquitous map information organization.

\subsection{Location-Based Aggregation Mode}

Location-based aggregation includes three core points that are closely related and mutually restricted: 1) It takes multi-modal and fragmented spatiotemporal ubiquitous information as the basic object it controls. 2) Its ultimate goal is to construct application scenes to meet users' needs. 3) Its fundamental basis is the semantic location that mainly includes entity information and relation information (Qi, Wang, Wang, \& Cui, 2014).

Focusing on the above three points, the task of location-based aggregation can be decomposed into three main links: element information extraction $\rightarrow$ association and fusion $\rightarrow$ scene construction. Element information extraction is to extract ubiquitous elements from the feature description of fragmented spatiotemporal ubiquitous information, as the information basis for aggregation computing. Association and fusion is the main computing link of location-based aggregation. Its main idea is to take location entities as the "condensation core", and associate and fuse spatiotemporal ubiquitous information based on the similarity of certain features. Scene construction is the further processing and presentation of the results of association and fusion.

The ubiquitous map can be a participant of location-based aggregation, providing "information material" for aggregation computing; and it can also be a carrier of location-based aggregation, providing a "display platform" for aggregation results. According to the specific needs of users, the available element information is extracted from the map scenes of the relevant theme, and the information is organically organized according to certain association relations and aggregation rules under the "location" framework to construct map scenes that meet users' needs.

\subsection{Map Scene}

Scenes are the link between ubiquitous maps and location-based aggregation. In this paper, the connotation of the ubiquitous map scene includes two aspects as "content scene" and "representation scene". The former is the inner meaning and the latter is the outer expression. The two aspects are interdependent and mapped to each other, forming a systematic and organic organization of ubiquitous map information. According to the location-based aggregation mode and the ubiquitous map information characteristics, the content and representa- 
tion scene information organization of ubiquitous maps can be divided into three levels as "features" $\leftrightarrow$ "elements" $\leftrightarrow$ "scenes" (Wang, Wang, \& Tian, 2021). As the basis of the map scene, the level of "features" describes the information form and content type that can be directly extracted from the ubiquitous map, and can adapt to the interpretation, organization, association and analysis of fragmented "micro-contents", forming a comprehensive and fine-grained description. The hierarchical construction from "features" to "elements" to "scenes" forms a systematic and complete ubiquitous map scene.

\subsection{Content Scene Organization}

The content scene reflects the intrinsic meaning of the ubiquitous map. It is a complex with a specific structure and function formed by the interconnection and interaction of various elements in different ranges in the ternary space, including three levels of "content features" $\leftrightarrow$ "content elements" $\leftrightarrow$ "content scenes":

- Level of "content features": Content features are the basis for multi-faceted reflection and description of the content of ternary space elements. Combined with related research (Lv, Yuan, \& Yu, 2017), this paper divides content features into six categories: temporal features, spatial features, geometric forms, attribute features, process features and semantic descriptions. Temporal features refer to the time nodes of the occurrence, development and demise of ternary space objects or phenomena, such as birth date and time of occurrence. Spatial features refer to the spatial nodes of the occurrence and development of ternary space objects or phenomena, such as place of occurrence, path, and scope of influence. Geometric forms refer to the composition and evolution structure of ternary space objects, such as geometric elements and shapes. Attribute features refer to the inherent properties of ternary space objects or phenomena, such as temperature and economic loss. Process features refer to the occurrence and evolution of ternary space objects or phenomena, such as temporal point and its state, time segment or behavior in the life cycle. Semantic features are ternary space features based on human cognition, such as name, type, subject and level.

- Level of "content elements": Content elements are the abstract expression of complex things and phenomena in the ternary space based on time-space, attributes, semantics, etc., involving objects and relations between elements. For the ternary space, content elements can be divided into six basic types: time, place, person, thing, event, and phenomenon (Lv, Yu, Yuan, Luo, Zhou, $\mathrm{Wu}$, et al., 2018). Each element type has independent connotation composition and meaning, and can be further subdivided according to its own characteristics. For example, the time element type can be further divided into absolute time, relative time and reference time; the person element type can be further divided into individual, group and organization. The element relation is a qualitative or quantitative relationship between elements based on 
space-time, geometry, attributes or semantics, mainly including temporal relation, spatial relation, attribute relation and compound relation.

- Level of "content scenes": There are various types of content scenes including spatial distribution, spatiotemporal evolution, event development, logical connection, etc. The content scene is composed of elements and element relations. Under the location-based aggregation mode, its organization idea is to associate and fuse "supporting role" elements with the "leading role" element as the core. The "leading role" corresponds to the "location entity", and the "supporting role" corresponds to the "element information".

\subsection{Representation Scene Organization}

The representation scene is a visual reflection of the content scene, and mainly refers to the scene graph or 3D scene as a graph or image, including three levels of "representation features" $\leftrightarrow \rightarrow$ "representation elements" $\leftrightarrow \rightarrow$ "representation scenes":

- Level of "representation features": Representation features are the reflection of content features, and are also the basis for describing and forming representation elements. The representation features of ubiquitous maps include visual variables, auditory variables, tactile variables, time variables, spatial variables, hierarchical variables, scale variables, virtual variables, interactive variables, etc., such as shape, size, color, sound, texture, lighting, shadow, duration, rate of change, etc.

- Level of "representation elements": Representation elements are the reflection of content elements, mainly involving map symbol objects, the relations between symbol objects and the structure of the map. Map symbols broadly cover point symbols, line symbols, polygon symbols, volume symbols, picture symbols, artistic symbols, color symbols, text annotations, node symbols, directed line symbols, etc. The relations between symbols include ordering relations, combination relations, topological relations, overlapping relations, etc. The map structure mainly includes map name, layer, legend, and map description. In the ubiquitous map, the representation forms of elements are flexible and diverse, that is, both abstract and concrete representation are allowed, and partial defaults are also allowed in the structure.

- Level of "representation scenes": A representation scene is composed of representation elements under a specific layout, pattern, configuration and style. It is a visual display of content scenes, such as $2 \mathrm{D}$ or $3 \mathrm{D}$ map, dynamic map, hand-painted map, etc.

\section{Example and Analysis}

Based on the theme of "Beijing Tourism", the application example of the ubiquitous map information organization model proposed in this paper is analyzed.

Focusing on the theme of "Beijing Tourism", relevant ubiquitous maps are collected from different websites and platforms (e.g. Sohu.com, Baidu.com, Sina 
Blog, "Zhihu" application platform, "Xiaohongshu" application platform, "xuexi.cn" learning platform), including Beijing metro roadmap, hand-painted map of local attractions, semantic map of Beijing attraction traffic time, Beijing tourist traffic map, 3D map of Beijing City, Beijing food map, distribution map of Beijing landmark buildings, urban humanistic map, etc. (see Figure 2).

According to the model proposed in this paper, the map scenes are decomposed step by step from the two aspects of content and representation, and various elements and features of attractions, food, architectures, topological associations, distance relations, time semantic associations, names, types, ranges, positions, shapes, properties and time can be acquired. The information can be used as basic resources to provide effective data support for information retrieval, aggregation applications and ubiquitous map construction on related topics. Parts of the elements and features are listed in Table 2 as an example.

Table 2. Hierarchical decomposition instances of map information.

\begin{tabular}{|c|c|c|c|}
\hline \multirow{2}{*}{\multicolumn{2}{|c|}{ Ubiquitous Map Scene }} & \multicolumn{2}{|c|}{ Element and Feature Information } \\
\hline & & Element instances & Feature description \\
\hline \multirow{3}{*}{$\begin{array}{l}\text { Beijing metro } \\
\text { roadmap }\end{array}$} & \multirow{3}{*}{$\begin{array}{l}\text { Content } \\
\text { representation }\end{array}$} & $\begin{array}{l}\text { Subway station: Guloudajie, Andingmen, } \\
\text { Yonghegong Lama Temple, ... }\end{array}$ & Station name, line number \\
\hline & & $\begin{array}{c}\text { Connectivity relation: } \\
\text { Guloudajie } \leftrightarrow \rightarrow \text { Andingmen, } \\
\text { Andingmen } \leftrightarrow \rightarrow \text { Yonghegong Lama Temple, } \ldots\end{array}$ & Station object, line name \\
\hline & & $\begin{array}{l}\text { Node, annotation } \\
\text { Connection line }\end{array}$ & $\begin{array}{l}\text { Form, size, character, color } \\
\text { Color, form, line type, size }\end{array}$ \\
\hline \multirow{4}{*}{$\begin{array}{l}\text { hand-painted } \\
\text { map of local attrac- } \\
\text { tions (Liulichang) }\end{array}$} & Content & $\begin{array}{c}\text { Architecture: Beijing Painting Shop, } \\
\text { Former Residence of Ji Xiaolan, China Bookstore, ... } \\
\text { Person: Ji Xiaolan }\end{array}$ & $\begin{array}{l}\text { Architecture name, } \\
\text { usage, owner, position } \\
\text { Name }\end{array}$ \\
\hline & \multirow{3}{*}{ representation } & $\begin{array}{c}\text { Place: Xuanwumen East St, Hufangqiao, Hepingmen, ... } \\
\text { Artistic symbol, annotation }\end{array}$ & $\begin{array}{c}\text { Name, range, direction } \\
\text { Color, pattern, texture, } \\
\text { form, size, shadow, character }\end{array}$ \\
\hline & & Annotation & Character \\
\hline & & Annotation, area symbol & Character, size, form \\
\hline \multirow{4}{*}{$\begin{array}{l}\text { semantic map of } \\
\text { Beijing attraction } \\
\quad \text { traffic time }\end{array}$} & \multirow[b]{4}{*}{ representation } & $\begin{array}{c}\text { Attraction: Palace Museum, Bird's Nest, } \\
\text { Water Cube, Beihai Park, Peking University, ... }\end{array}$ & Attraction name \\
\hline & & $\begin{array}{c}\text { Temporal relation: Palace Museum } \rightarrow \text { Bird's } \\
\text { Nest and Water Cube, Palace Museum } \rightarrow \text { Beihai Park, } \\
\text { Palace Museum } \rightarrow \text { Peking University, ... }\end{array}$ & $\begin{array}{l}\text { Attraction object, duration } \\
\text { driven by events }\end{array}$ \\
\hline & & Point symbol, annotation & Form, color, size, character \\
\hline & & Directional line symbol, annotation & $\begin{array}{l}\text { Line type, line color, } \\
\text { line direction, character }\end{array}$ \\
\hline \multirow{2}{*}{$\begin{array}{l}\text { 3D map of } \\
\text { Beijing City }\end{array}$} & content & Architecture, green space, water & Position \\
\hline & representation & 3D symbol & Texture, Color, shadow, form \\
\hline
\end{tabular}




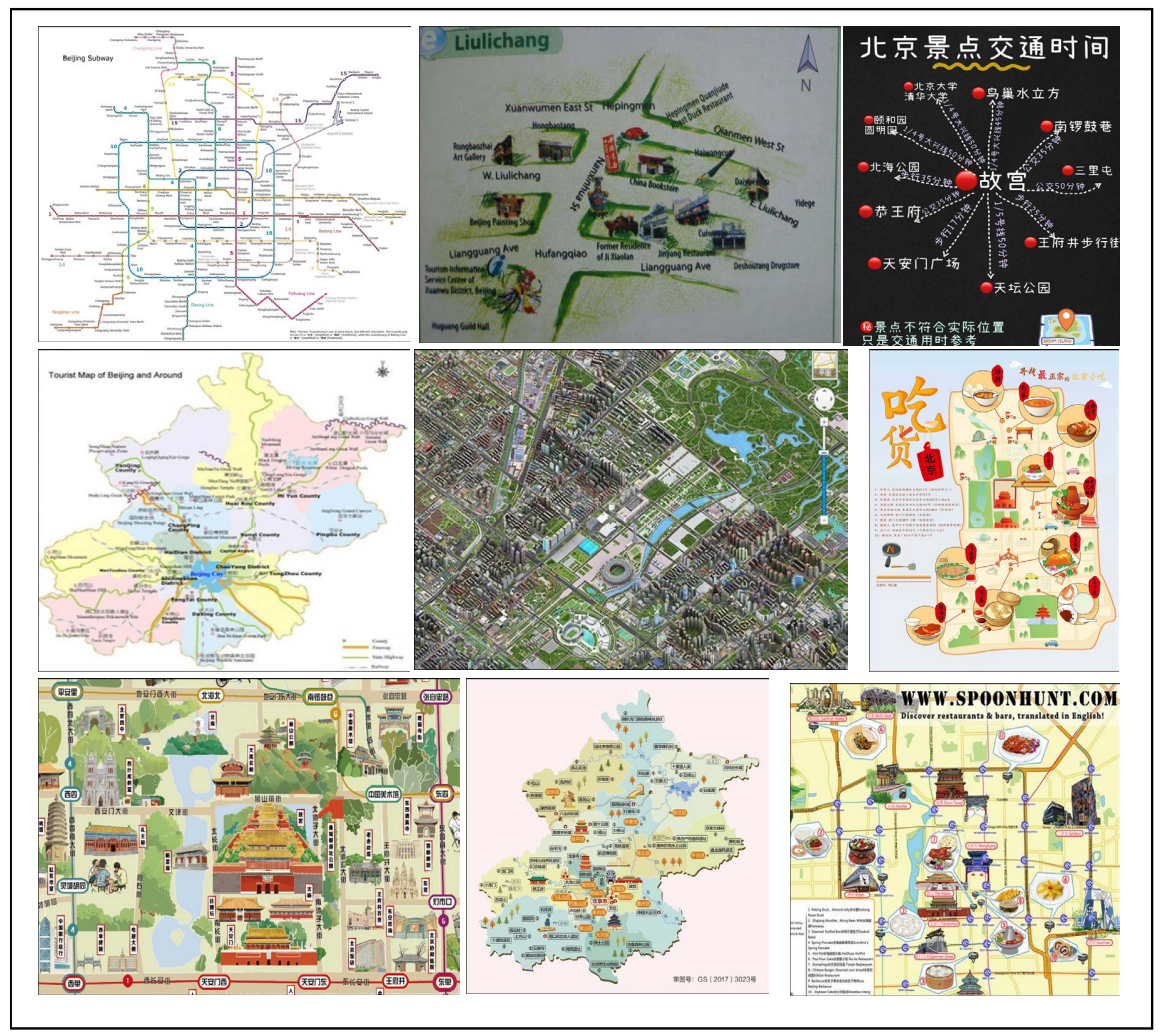

Figure 2. Ubiquitous map instances with the theme of "Beijing Tourism".

\section{Conclusion}

In the era of ICT and big data, with the expansion of the object and expression space of maps from the dual space of "geography-humanity" to the ternary space of "geography-humanity-information", and the expansion of information sources from standard geographic information to fragmented and multi-modal spatiotemporal ubiquitous information, the concept of "ubiquitous map" came into being, which puts forward new and complex requirements for information organization. Location-based aggregation is an effective solution to solve the contradiction between massive spatiotemporal ubiquitous information and complex information needs of users in the era of ICT and big data, providing accurate location-related knowledge services. It can provide an effective mode for ubiquitous map information organization. This paper introduces the idea of "location-based aggregation" into ubiquitous map information organization, establishes a ubiquitous map information organization model, and improves the information organization mechanism of ubiquitous maps from two aspects as internal content and external representation. The model proposed in this paper can achieve a complete coverage of the ternary space and a fine-grained semantic description of the fragmented spatiotemporal ubiquitous information, providing effective support for the automatic construction of ubiquitous maps and information applications. In the future, the experimental verification and practical application of this model are expected to be further enriched. 


\section{Conflicts of Interest}

The authors declare no conflicts of interest regarding the publication of this paper.

\section{References}

Ai, T. H. (2016). Development of Cartography Driven by Big Data. Journal of Geomatics, $41,1-7$.

Cao, S. J., \& Ma, C. C. (2016). Information Aggregation: Concept and Paradigms. Journal of Library Science in China, 42, 4-19.

Guo, R. Z., \& Ying, S. (2017). The Rejuvenation of Cartography in ICT Era. Acta Geodaetica et Cartographica Sinica, 46, 1274-1283.

Guo, R. Z., Chen, Y. B., Ying, S., Lv, G. N., \& Li, Z. L. (2018). Geographic Visualization of Pan-Map with the Context of Ternary Space. Geomatics and Information Science of Wuhan University, 43, 1603-1610.

Kresse, W., \& Danko, D. M. (2012). Springer Handbook of Geographic Information. Springer. https://doi.org/10.1007/978-3-540-72680-7

Liu, J. N., Fang, Y., Guo, C., \& Gao, K. F. (2014). Research Progress in Location Big Bata Analysis and Processing. Geomatics and Information Science of Wuhan University, 39, 379-385.

Liu, J. N., Guo, W. F., Guo, C., Gao, K. F., \& Cui, J. S. (2020). Rethinking Ubiquitous Mapping in the Intelligent Age. Acta Geodaetica et Cartographica Sinica, 49, 403-414.

Liu, Z., \& Li, Z. L. (2016). Impact of Schematic Designs on the Cognition of Underground Tube Maps. ISPRS: International Archives of the Photogrammetry, Remote Sensing and Spatial Information Sciences, XLI-B2, 421-423. https://doi.org/10.5194/isprsarchives-XLI-B2-421-2016

Lv, G. N., Yu, Z. Y., Yuan, L. W., Luo, W., Zhou, L. C., Wu, M. G. et al. (2018). Is the Future of Cartography the Scenario Science?. Journal of Geo-information Science, 20, 1-6.

Lv, G. N., Yuan, L. W., \& Yu, Z. Y. (2017). Surveying and Mapping Geographical Information from the Perspective of Geography. Acta Geodaetica et Cartographica Sinica, 46, 1549-1556.

Meng, L. Q. (2017). The Constancy and Volatility in Cartography. Acta Geodaetica et Cartographica Sinica, 46, 1637-1644.

Qi, X. F., Wang, G. X., Wang, F. Q., \& Cui, X. F. (2014). Research of Semantic Location Modeling Based on Location Service. Science of Surveying and Mapping, 39, 89-92.

Wang, J. Y. (2017). Cartography in the Age of Spatio-temporal Big Data. Acta Geodaetica et Cartographica Sinica, 46, 1226-1237.

Wang, S., Wang, G. X., \& Tian, J. P. (2021). Classification Model of Ubiquitous Map Information Facing Location-Based Aggregation. Acta Geodaetica et Cartographica Sinica, 50, 789-799.

Yan, H. W., Zhang, L. M., Du, P., Liu, T., Wang, Z. H., \& Yang, W. F. (2016). We Map: A New Type of Map in the Era of We Media. Journal of Geomatics Science and Technology, 33, 520-523.

Zhou, C. H. (2015). Prospects on Pan-Spatial Information System. Progress in Geography, 34, 129-131.

Zhou, C. H., Zhu, X. Y., Wang, M., Shi, C., \& Ou, Y. (2011). Panoramic Location-Based Map. Progress in Geography, 30, 1331-1335. 
Zhu, X. Y., Zhou, C. H., Guo, W., Hu, T., Liu, H. Q., \& Gao, W. X. (2015). Preliminary Study on Conception and Key Technologies of the Location-Based Pan-Information Map. Geomatics and Information Science of Wuhan University, 40, 285-295. 